\title{
DNA index and karyotype analysis in myelodysplasia
}

\author{
T G HOY, A D GEDDES, A JACOBS \\ From the Department of Haematology, University of Wales College of Medicine, Cardiff
}

SUMMARY DNA index (DI) determined by flow cytometry and karyotype determined by conventional methods were obtained on bone marrow samples from 43 haematologically normal subjects and 54 patients with myelodysplastic syndrome (MDS). Twenty one patients had a clonal karyotype abnormality but an additional five had a DI outside the normal range, showing evidence of aneuploidy that was not available from chromsome preparations. When patients were grouped into those with excess chromosomal material, those with diploid karyotypes, and those with a loss of chromosomal material, there was a significant difference among the mean DIs of each group, normal subjects being different from all patient groups. In these patients DI measurements were of value when carried out together with conventional chromsomal analysis in gaining the maximum amount of genetic information when a satisfactory karyotype might not be available or where failure of an abnormal cell population to proliferate might give an incomplete cytogenetic picture. The contribution of non-clonal chromsome loss to the DI is probably significant but has not been quantitated.

Cytogenetic abnormalities are common in patients with the myelodysplastic syndrome (MDS) and more complex abnormalities are associated with a poor prognosis. ${ }^{1}$ Heim and Mitelman pointed out that small abnormal clones may remain undetected in cytogenetic analysis. ${ }^{2}$ It is not always possible, however, to evaluate accurately the prevalence and extent of karyotype abnormalities because of poor chromosome morphology and the tendency for abnormal clones to proliferate poorly. ${ }^{3}$ The measurement of cellular DNA content by flow cytometry provides an alternative method for estimating ploidy that is independent of cell proliferation and has been exploited for this purpose in patients with acute lymphoblastic leukaemia, ${ }^{45}$ myelomatosis, ${ }^{6}$ adult acute leukaemia ${ }^{7}$ and myelodysplastic syndrome. ${ }^{89}$ Although the DNA content of marrow cells in all these conditions has been related to prognosis, it has not always been clear to what extent these measurements reflect clonal karyotype abnormalities, random chromosome loss, or variations in the proportion of abnormal cells in the marrow. In this study we compared the presence of aneuploidy defined by conventional karyotype analysis with the DNA index (DI) derived from flow cytometric analysis of bone marrow cells in 54 cases of MDS and 43 haematologically normal subjects.

Accepted for publication 12 January 1989

\section{Material and methods}

Fifty four patients with MDS as defined by the FAB criteria ${ }^{10}$ modified by May et $a l^{11}$ were included in this study, together with 43 haematologically normal subjects undergoing cardiac surgery. The protocols were approved by the South Glamorgan joint ethical committee. In the case of patients bone marrow aspirates were used for analysis and in surgical patients marrow samples were obtained from the exposed surface of the sternum during the operation.

Bone marrow aspirates from patients with MDS were placed in $10 \mathrm{ml}$ RPMI 1640 medium (Gibco) containing $15 \%$ fetal calf serum (Gibco) at a concentration of $1-1.5 \times 10^{7}$ nucleated cells $/ \mathrm{ml}$. The cultures were incubated for two hours in the presence of colcemid $(1 \mu \mathrm{g} / \mathrm{ml})$, for 24 hours with colcemid present, from three-24 hours and for 24 hours with colcemid present for one hour before harvesting. Hypotonic treatment consisted of $0.075 \mathrm{M}$ potassium chloride, for 20 minutes. Fixation comprised three changes of methanol:glacial acetic acid $(3: 1)$, overnight storage of the cells in fixative at $-20^{\circ} \mathrm{C}$, followed by a further wash in fixative. Slides were prepared and examined using trypsin/Leishmann (GTL) banding. Slides from each culture were assessed and where possible analysed. A minimum of five G-banded metaphases from two or more cultures was necessary for a successful analysis. Where possible, at least 30 
metaphases were counted and 20 fully karyotyped by G-banding. Chromosome abnormalities were defined as clonal by the accepted criteria - that is, two or more metaphases containing the same additional chromosome or structural rearrangement and three or more metaphases showing loss of the same chromosome. A karyotype was only conclusively reported as normal where full analysis of at least 20 metaphases was possible.

Several authors have calculated a karyotype index based on the mean chromsome number per metaphase. ${ }^{12-14}$ We decided not to use this variable because of the large variations arising from the analysis of too few metaphases and the random loss of chromosomes during processing. Karyotypes from patients were classified as diploid where only normal metaphases were found, pseudo/hypodiploid where a clonal abnormality characterised by partial or complete chromsome loss was seen, and pseudo/hyperdiploid when there was a clonal abnormality due to additional chromosomal material.

Marrow samples from all subjects were prepared and stained as in previous studies. ${ }^{8}$ Nuclei were stained with propidium iodide following the protocol of Vindelov ${ }^{15}$ and analysed on a FACSIII (Becton Dickinson) using $488 \mathrm{~mm}$ excitation wavelength and analysing through a $620 \mathrm{~nm}$ long pass filter. The DI as defined by Barlogie et al $^{13}$ was calculated on duplicate samples of 10000 cells. Chicken and trout erythrocyte nuclei were used as internal standards and human peripheral blood lymphocyte nuclei were used as a reference standard to minimise interbatch variation. ${ }^{16}$ The use of a high resolution staining technique in conjunction with two internal and one external standard permitted a high degree of resolution and reproducibility. Differences in DNA content of male and female cells $(1.78 \%)$ are easily detected and a correction factor of 0.9825 was applied to female subjects.

The DI of the normal marrow samples was compared with those of the diploid, pseudo/hypodiploid, and pseudo/hyperdiploid patient marrows using the Mann-Whitney U test.

\section{Results}

The sensitivity of the method is shown in fig 1 . Histograms of normal male and female mononuclear cells were superimposed after normalising the fluorescence scale using the internal reference standards. The difference in diploid DNA content is clearly visible. The mean position for 10 men and 10 women was used to determine the difference $(1 \cdot 78 \%)$.

The mean DI for normal subjects was 1.004 (SD 0.005). The DI for the cytogenetically different groups of patients is shown in the table. The different groups

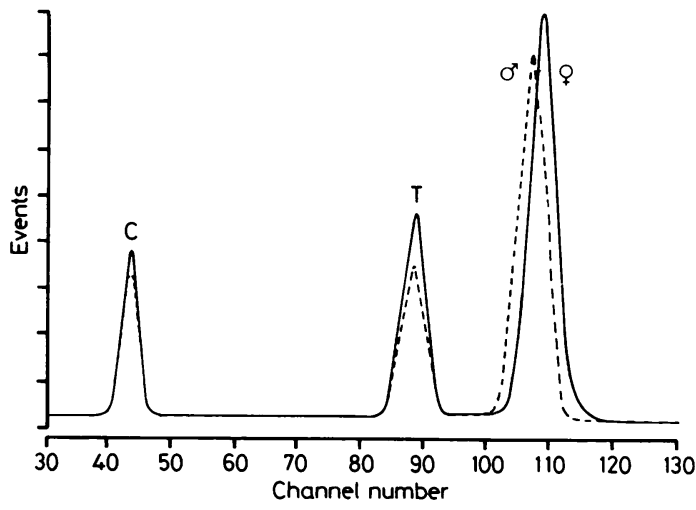

Fig 1 DNA histograms of a normal man and a normal woman, each containing chicken $(C)$ and trout $(T)$ erythrocyte nuclei as internal reference standards. The fluorescence scales have been normalised to superimpose the chicken and trout peaks.

of patients were significantly different from each other, those with a pseudo/hyperdiploid karyotype having a higher $(p=0.0039)$ and those with a pseudo/hypodiploid karyotype having a lower $(p=0.0008)$ DI than diploid patients. The mean DI of patients with a diploid karyotype was significantly lower than that of normal subjects $(p=0.006)$. Thirty three of the patients with myelodysplasia had a pseudo/diploid karyotype on cytogenetic analysis but of these, five had a DI outside the range of normal results (fig 2). Four of 10 patients with an excess of chromosomal material had a DI higher than that of normal subjects and three of 11 patients showing loss of chromsome material had a DI less than that of normal subjects. In total, 21 of 54 patients had a clonal karyotype abnormality, of whom seven had an abnormal DI, and 12 of the 54 patients had a DI outside the normal range, seven of whom had a clonal karyotype abnormality. Twenty six patients had either one or both abnormalities.

Among the pseudo/hyperdiploid group, two of four patients with a high DI had a constitutional 47,XXY and $47, X X X$ karyotype, respectively. In one all meta-

Table Mean DNA index in normal subjects and patients with myelodysplastic syndrome

\begin{tabular}{|c|c|c|c|c|c|}
\hline & $\begin{array}{l}\text { DNA } \\
\text { index }\end{array}$ & $S D$ & & p Value & \\
\hline $\begin{array}{l}\text { Normal subjects } \\
\text { Patients }\end{array}$ & 1.004 & 0.005 & \multirow[t]{2}{*}{0.006} & \multirow{4}{*}{$\begin{array}{r}] \\
0.0001\end{array}$} & \multirow{4}{*}{$\begin{array}{l}0.039 \\
0.0008\end{array}$} \\
\hline Pseudo/hyperdiploid & $1 \cdot 013$ & 0.014 & & & \\
\hline Diploid & 1.000 & 0.008 & & & \\
\hline Pseudo/hypodiploid & 0.990 & 0.010 & & & \\
\hline
\end{tabular}




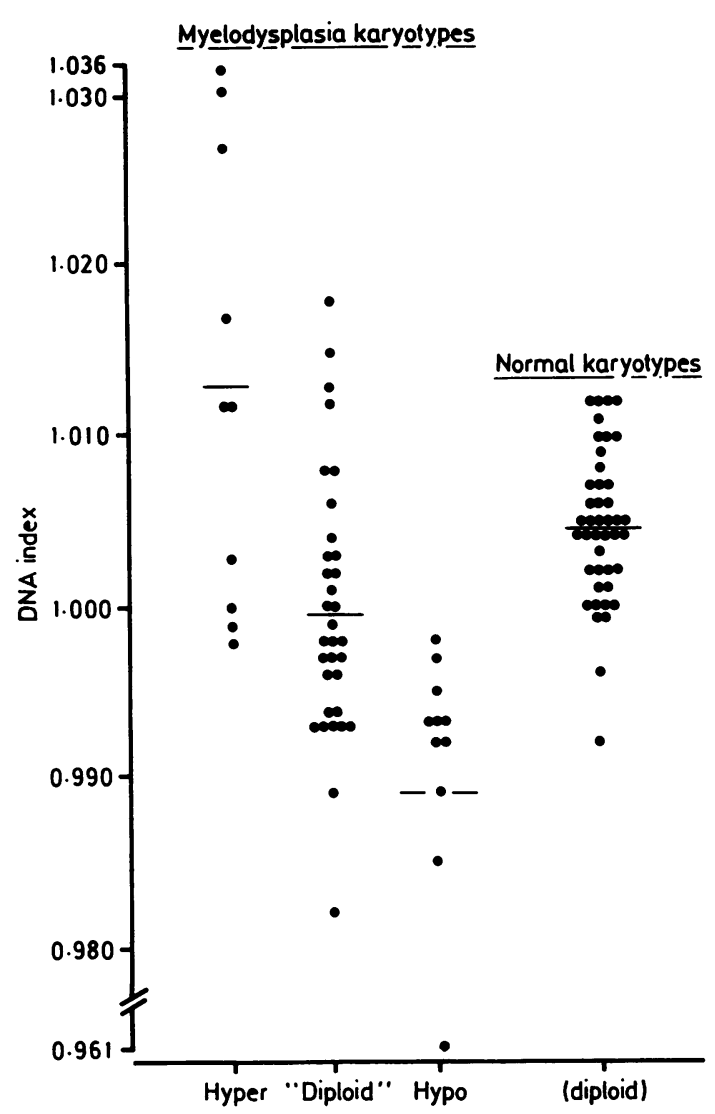

Fig 2 DNA index of normal marrow samples compared with those from myelodysplastic patients subdivided into those with pseudo/hyperdiploid (hyper) or pseudo/hypodiploid (hypo) clonal karyotype abnormalities and those with a diploid karyotype. Mean values indicated by horizontal lines.

phases showed trisomy 8 , and in one a clone with +10 , $+19,+21$ was observed. The four patients with the lowest DI in this group, however, all of which were within the normal range, included three patients in whom a clone with trisomy 8 comprised $50 \%, 70 \%$, and $100 \%$ of the metaphases examined, and one patient with an additional marker chromosome, derived from a deleted and rearranged chromsome 5, roughly the size of chromsome 16 . This last abnormality would have been below the limit of detection by flow cytometry but the DNA histogram of the three patients with trisomy 8 all showed distortion of the $\mathrm{G}_{0} / \mathrm{G}_{1}$ peak.

Among the hypodiploid group, most clonal karyotype abnormalities consisted of partial deletions including 5q-. 7q-. 11q- and 12q-, the only whole chromosome loss being that of $\mathrm{Y}$ in one case. Nonclonal chromosome loss not apparently resulting from technical artefact was common in metaphases from all patient groups and was particularly pronounced in some persons.

\section{Discussion}

Hoehn et al have shown that well controlled flow cytometry can resolve subtle degrees of difference in the genome size of cultivated human cell lines,,${ }^{17}$ and we have previously shown that it is possible to demonstrate similar differences in DI in human bone marrow samples. ${ }^{89}$ It is usually assumed that there is a good correlation between DI and the chromsomal composition of the cells and this has been shown in several instances. ${ }^{1318}$ The findings of an earlier study have shown that in patients with MDS the DI of bone marrow cells is a powerful indicator of prognosis, those with a low DI having a much shorter survival than others. ${ }^{9}$ It was assumed that in this condition, too, the phenomenon was related to chromsome loss.

The presence of clonal karyotype abnormalities in $38.9 \%$ of the 54 patients studied here is similar to the incidence found by most other workers except Yunis et al. ${ }^{19}$ Nowell et al found $43 \%$ of cases to have a clonal abnormality, ${ }^{1}$ Jacobs et al $39 \%,{ }^{20}$ Kerndrup et al $41 \%,{ }^{21}$ and Weh et al $48 \%,{ }^{22}$ the higher incidence in the last series being partly due to the inclusion of patients with previous exposure to carcinogenic agents. The present study suggests that clonal ploidy abnormalities are an important factor in determining DI in patients with MDS.

There was a significant difference between the DI of diploid, pseudo/hyperdiploid, and psuedo/hypodiploid marrow samples. Although there was overlap between the range of values in the three groups, this was minimal between the pseudo/hyperdiploid and pseudo/hypodiploid groups $(\mathrm{p}=0.0001)$. The mean DI of samples from patients with no demonstrable clonal chromosome abnormality, however, was $0.4 \%$ lower than that of normal subjects (fig 2). In addition to the lowering of DI in the diploid group compared with normal subjects, patients in the pseudo/hyperdiploid group often had a DI in the normal range despite the presence of an additional chromsome. While the reason for these phenomena cannot be determined with certainty from the present data, non-clonal chromosome loss, not obviously attributable to artefact, was commonly observed in patient tissue and this may well be an important factor. Differences in the staining of DNA from diseased marrow cannot be excluded, nor can the presence of non-dividing hypodiploid cell populations. While karyotype analysis in myelodysplastic marrows is commonly based on examination of a relatively low number of metaphases, often selected for their good morphology, ${ }^{2}$ the DNA content of marrow cells based on 
the measurement of 10000 nuclei is far more representative of the total population, though it is less sensitive to minor degrees of DNA loss and cannot detect balanced translocations.

In the present series 21 of 54 patients were found to have a clonal karyotype abnormality but an additional five cases had a DI outside the range found in normal subjects, providing evidence of aneuploidy that was not obtainable from the chromosome preparations. Eight further cases were not included in the present analysis as insufficient metaphases were available for karyotype analysis. Of these, five had a normal DI; in two the DI was low and in one it was high. In both patients with a low DI karyotype analysis carried out on other occasions showed abnormal clones. Poor proliferation of haemopoietic cells is often found in marrow cultures from patients with MDS, and Barlogie et al have pointed out that under such circumstances the use of DNA cytometry can give an indication of ploidy independent of proliferative activity. ${ }^{67}$ During the period of this study DI measurements were made on marrow samples from an additional 41 patients with MDS in whom chromosome preparations were not made. Twenty eight of these had a normal DI, in six the DI was low, and in seven it was abnormally high. Smets et al, reviewing their study of childhood acute lymphoblastic leukaemia, concluded that the two techniques of chromosome analysis and flow cytometry analysis of DNA should be used jointly, to obtain the maximum amount of genetic information on the leukaemic cell population. ${ }^{5}$ Barlogie et al came to a similar conclusion with regard to patients with adult acute leukaemia and pointed out that the two techniques are complementary.? Our own experience, showing the importance of DNA hypoploidy in predicting prognosis, ${ }^{9}$ and the present data comparing DNA ploidy and chromsome analysis, lead us to draw the same conclusion for patients with MDS.

\section{References}

1 Nowell PC, Besa EC, Stelmach T, Finan JB. Chromosome studies in preleukaemic states. Cancer 1986;58:2571-5.

2 Heim S, Mitleman F. Chromosome abnormalities in the myelodysplastic syndromes. Clin Haematol 1986;15:1003-21.

3 Nowell PC. Cytogenetics of preleukaemia. Cancer Genet Cytogenet 1982;5:265-78.

4 Look AT, Robertson PK, Williams DL, et al. Prognostic importance of blast cell DNA content in childhood acute lymphoblastic leukemia. Blood 1985;65:1079-86.

5 Smets LA, Slater R, Behrendt H, Van't Veer MB, Holman-Blok J.
Phenotypic and karyotypic properties of hyperdiploid acute lymphoblastic leukaemia of childhood. $\mathrm{Br} J$ Haematol 1985;61:113-23.

6 Barlogie B, Alexanian R, Dixon D, Smith L, Smallwood L, Delasalle K. Prognostic implications of tumor cell DNA and RNA content in multiple myeloma. Blood 1985;66:338-41.

7 Barlogie B, Stass C, Dixon D, et al. DNA aneuploidy in adult acute leukaemia. Cancer Genet Cytogenet 1987;28:213-28.

8 Peters SW, Clark RE, Hoy TG, Jacobs A. DNA content and cell cycle analysis of bone marrow cells in myelodysplastic syndromes (MDS). Br J Haematol 1986;62:239-46.

9 Clark R, Peters S, Hoy T, Smith S, Whittaker K, Jacobs A. Prognostic significance of hypodiploid haemopoietic precursors in myelodysplastic syndromes. $N$ Engl J Med 1986;314:1472-5.

10 Bennett JM, Catovsky D, Daniel MT, et al. Proposals for the classification of the myelodysplastic syndromes. $\mathrm{Br} \mathrm{J}$ Haematol 1982;51:189-99.

11 May SJ, Smith SA, Jacobs A, Williams A, Bailey-Wood R. The myelodysplastic syndrome: analysis of laboratory characteristics in relation to the FAB classification. $B r J$ Haematol 1985;59:311-9.

12 Barlogie B, Hittleman W, Spitzer G, et al. Correlation of DNA distribution abnormalities with cytogenetic findings in adult leukaemia and lymphoma. Cancer Res 1977;37:4400-7.

13 Barlogie B, Raber MN, Schumann J, et al. Flow cytometry in clinical cancer research. Cancer Res 1983;43:3982-97.

14 Riccardi A, Montecucco CM, Danova M, et al. Flow cytometric evaluation of proliferative activity and ploidy in myelodysplastic syndromes and acute leukaemias. Basic and Applied Histochemistry 1986;30:181-92.

15 Vindelov LL, Christensen IJ, Nissen NI. A detergent-trypsin method for the preparation of nuclei for flow cytometric DNA analysis. Cytometry 1983;3:323-7.

16 Jakobsen A. The use of trout erythrocytes and human lymphocytes for standardisation in flow cytometry. Cytometry 1983; 4:161-5.

17 Hoehn H, Koch H, Kohler J, et al. Interphase cell flow cytometry as a means of monitoring genomic size in normal and neoplastoid cell cultures. Cancer Genet Cytogenet 1987;24:191-204.

18 Andreef M, Conjalka M, Jhanwar S, et al. Clonal abnormalities in acute lymphoblastic leukaemia: comparison of cytogenetics and flow cytometry. Blood 1982;60(suppl)120a.

19 Yunis JJ, Rydell RE, Oken MM, Arnesen MA, Mayer MG, Lobell $M$. Refined chromosome analysis as an independent prognostic indicator in de novo myelodysplastic syndromes. Blood 1986;67:1721-30.

20 Jacobs RH, Cornbleet MA, Vardiman JW, Larson RA, Le Beau MM, Rowley JD. Prognostic implications of morphology and karyotype in primary myelodysplastic syndromes. Blood 1986;67:1765-72.

21 Kerndrup G, Bendix-Hansen K, Pedersen B. The prognostic significance of cytological, histological and cytogenic findings in refractory anaemia (RA) and RA with sideroblasts. A follow up study. Blut 1987;54:231-8.

22 Weh HJ, Calavrezos A, Seeger D, Kuse R, Horsfield DK. Cytogenetic studies in 69 patients with myelodysplastic syndromes (MDS). Eur J Haematol 1987;38:166-72.

Requests for reprints to: Professor A Jacobs, Department of Haematology, University of Wales College of Medicine, Heath Park, Cardiff CF4 4XN, Wales. 